Publ. Mat. (2014), 49-62

Proceedings of New Trends in Dynamical Systems. Salou, 2012.

DOI: $10.5565 /$ PUBLMAT_Extra14_03

\title{
IRREGULAR SETS FOR RATIOS OF BIRKHOFF AVERAGES ARE RESIDUAL
}

\author{
Luis Barreira, Jinjun Li, and Claudia Valls
}

Dedicated to Jaume Llibre on the occasion of his 60th birthday

\begin{abstract}
It follows from Birkhoff's Ergodic Theorem that the irregular set of points for which the Birkhoff averages of a given continuous function diverge has zero measure with respect to any finite invariant measure. In strong contrast, for systems with the weak specification property, we show here that if the irregular set is nonempty, then it is residual. This includes topologically transitive topological Markov chains, sofic shifts and more generally shifts with the specification property. We consider also the more general case of ratios of Birkhoff averages of continuous functions and the case when the set of accumulation points of the ratios of Birkhoff averages is a prescribed closed interval. Finally, we give an application of our work to the pointwise dimension of a Gibbs measure on a repeller of a conformal map.
\end{abstract}

2010 Mathematics Subject Classification: Primary: 37B10.

Key words: Birkhoff averages, irregular sets, weak specification.

\section{Introduction}

Given a continuous map $f: X \rightarrow X$ on a compact metric space, the irregular set for a function $\varphi: X \rightarrow \mathbb{R}$ is defined by

$$
X_{\varphi}=\left\{x \in X: \liminf _{n \rightarrow \infty} \frac{1}{n} \sum_{i=0}^{n-1} \varphi\left(f^{i}(x)\right)<\limsup _{n \rightarrow \infty} \frac{1}{n} \sum_{i=0}^{n-1} \varphi\left(f^{i}(x)\right)\right\} .
$$

By Birkhoff's Ergodic Theorem, the set $X_{\varphi}$ has zero measure with respect to any finite $f$-invariant measure on $X$. On the other hand, it was shown in [4] that from the point of view of dimension theory the set $X_{\varphi}$ can be as large as the whole space. This phenomenon was first observed by Pesin and Pitskel' in [9] for the full shift on two symbols. We refer the

Partially supported by FCT (grant PTDC/MAT/117106/2010 and CAMGSD). Jinjun Li was also supported by the National Natural Science Foundation of China (grant no. 11071082) and the Education Committee of Fujian Province (grant no. JA11173). 
reader to the book $[\mathbf{1}]$ for a detailed discussion and to $[\mathbf{3}, \mathbf{5}, \mathbf{6}, \mathbf{7}, \mathbf{8}, \mathbf{1 0}]$ for further related work.

For systems with the weak specification property, we show here that the irregular set $X_{\varphi}$ for a continuous function is either empty or residual. This includes topologically transitive topological Markov chains, sofic shifts and more generally shifts with the specification property. More generally, we consider ratios of Birkhoff averages and we show that some subsets of the irregular set are also residual. Namely, given continuous functions $\varphi, \psi: X \rightarrow \mathbb{R}$ with $\inf \psi>0$ and an interval $I \subset \mathbb{R}$, let

$$
X_{I}=\{x \in X: A(x)=I\},
$$

where $A(x)$ is the set of accumulation points of the sequence

$$
S_{\varphi, \psi}(x, n)=\frac{\sum_{i=0}^{n-1} \varphi\left(f^{i}(x)\right)}{\sum_{i=0}^{n-1} \psi\left(f^{i}(x)\right)} .
$$

In order to show that the set $X_{I}$ is residual we bridge together strings of sufficiently large length corresponding to Birkhoff averages with different limits in the interval $I$, proceeding in a similar manner to that in [2] where we have considered earlier the simpler case when $\psi=1$.

Now we give an application of our work to the dimension of repellers of conformal maps (we refer the reader to the book [1] for a detailed introduction to the area). Let $f: M \rightarrow M$ be a $C^{1}$ map on a smooth manifold and let $J \subset M$ be a compact $f$-invariant set. We say that $f$ is expanding on $J$ and that $J$ is a repeller for $f$ if there exist $c>0$ and $\tau>1$ such that

$$
\left\|d_{x} f^{n} v\right\| \geq c \tau^{n}\|v\|
$$

for $x \in J, v \in T_{x} M$ and $n \in \mathbb{N}$. Moreover, we assume that $f$ is topologically mixing and conformal on $J$ (we recall that $f$ is said to be conformal on a set $J$ if $d_{x} f$ is a multiple of an isometry for every $\left.x \in J\right)$.

One can use Markov partitions and the associated symbolic dynamics to transfer the results for symbolic dynamics (now for topologically mixing topological Markov chains) to results for repellers (see [1, 2] for details). In particular, if $\varphi$ is a Hölder continuous potential of a Gibbs measure $\mu$ on $J$ and $\psi=\log \|d f\|$, then

(1) $\lim _{n \rightarrow \infty} S_{-\varphi, \psi}(x, n)=\lim _{n \rightarrow \infty}-\frac{\sum_{i=0}^{n-1} \varphi\left(f^{i}(x)\right)}{\log \left\|d_{x} f^{n}\right\|}=\lim _{r \rightarrow 0} \frac{\log \mu(B(x, r))}{\log r}$ 
whenever any of the limits exist (assuming without loss of generality that $\varphi$ has zero topological pressure). The limit

$$
d_{\mu}(x)=\lim _{r \rightarrow 0} \frac{\log \mu(B(x, r))}{\log r},
$$

when it exists, is called the pointwise dimension of $\mu$ at $x$. The following result is a simple consequence of our main Theorem 3 together with (1).

Theorem 1. Let $J$ be a repeller of a $C^{1}$ map $f$ that is conformal and topologically mixing on J. Given a Gibbs measure $\mu$ on $J$ with a Hölder continuous potential and a closed interval

$$
I \subset\left(\inf _{\nu} \frac{\int_{X} \varphi d \nu}{\int_{X} \psi d \nu}, \sup _{\nu} \frac{\int_{X} \varphi d \nu}{\int_{X} \psi d \nu}\right)
$$

that is not a singleton, with the infimum and supremum taken over all $f$-invariant probability measures on $J$, the set $X_{I}$ is either empty or residual.

A simple consequence of this result is the following.

Theorem 2. Let $J$ be a repeller of a $C^{1}$ map $f$ that is conformal and topologically mixing on J. If $\mu$ is a Gibbs measure with a Hölder continuous potential, then

$$
\left\{x \in J: \liminf _{r \rightarrow 0} \frac{\log \mu(B(x, r))}{\log r}<\limsup _{r \rightarrow 0} \frac{\log \mu(B(x, r))}{\log r}\right\}
$$

is either empty or residual.

The proofs of Theorems 1 and 2 follow closely corresponding arguments in [2] for symbolic systems and thus are omitted.

We emphasize that quite the opposite happens from the point of view of ergodic theory: for large classes of dynamical systems and measures (such as any hyperbolic measure invariant under a $C^{1+\alpha}$ diffeomorphism), the pointwise dimension $d_{\mu}(x)$ is well defined for $\mu$-almost every $x$. For example, in the context of Theorem 1 this is a simple consequence of Birkhoff's Ergodic Theorem together with (1). On the other hand, it was shown in [4] that for several classes of dynamical systems and measures the set of points for which $d_{\mu}(x)$ is not well defined, if nonempty, has full topological entropy and full Hausdorff dimension. The present work shows that this set can also be as large as the whole space from the point of view of topology. 


\section{Residual irregular sets}

2.1. Basic notions. Let $\sigma: \Sigma^{+} \rightarrow \Sigma^{+}$be the shift map on the space of one-sided sequences $\Sigma^{+}=\{1, \ldots, k\}^{\mathbb{N}}$, where $k \geq 2$ is an integer. We equip $\Sigma^{+}$with the distance

$$
d\left(\omega, \omega^{\prime}\right)=2^{-n}, \quad \omega=\left(\omega_{i}\right)_{i \in \mathbb{N}}, \omega^{\prime}=\left(\omega_{i}^{\prime}\right)_{i \in \mathbb{N}},
$$

where $n$ is the smallest integer such that $\omega_{n} \neq \omega_{n}^{\prime}$.

Given a compact set $X \subset \Sigma^{+}$such that $\sigma(X) \subset X$, we consider the subshift $\sigma \mid X: X \rightarrow X$.

For each $n \in \mathbb{N}$, let

$$
X_{n}=\left\{\left(\omega_{1} \cdots \omega_{n}\right):\left(\omega_{1} \omega_{2} \cdots\right) \in X\right\} \quad \text { and } \quad X^{*}=\bigcup_{n \in \mathbb{N}} X_{n} .
$$

When $\omega=\left(\omega_{1} \omega_{2} \cdots\right) \in X$ and $m \in \mathbb{N}$ or when $\omega=\left(\omega_{1} \cdots \omega_{n}\right) \in X_{n}$ and $m \in \mathbb{N}$ with $m \leq n$, we write $\omega \mid m=\omega_{1} \cdots \omega_{M}$.

Moreover, for each $\omega \in X_{n}$, we write $|\omega|=n$ and

$$
[\omega]=\{\rho \in X: \rho \mid n=\omega\} .
$$

Given $\omega=\left(\omega_{1} \cdots \omega_{n}\right) \in X_{n}$ and $\omega^{\prime}=\left(\omega_{1}^{\prime} \cdots \omega_{m}^{\prime}\right) \in X_{m}$, let

$$
\omega \omega^{\prime}=\left(\omega_{1} \cdots \omega_{n} \omega_{1}^{\prime} \cdots \omega_{m}^{\prime}\right) .
$$

We note that, in general, $\omega \omega^{\prime}$ need not belong to $X^{n+m}$. We say that a subshift $\sigma \mid X$ has the weak specification property if there exists $\tau \in \mathbb{N} \cup\{0\}$ such that for each $\omega, \omega^{\prime} \in X^{*}$ there exists

$$
\rho=\rho\left(\omega, \omega^{\prime}\right) \in \bigcup_{k=0}^{\tau} X_{k}
$$

such that $\omega \rho \omega^{\prime} \in X^{*}$. The string $\rho\left(\omega, \omega^{\prime}\right)$ is called a bridge between $\omega$ and $\omega^{\prime}$. We shall write $\omega \rho \omega^{\prime}=\omega \bowtie \omega^{\prime}$ (although we emphasize that $\rho$ need not be unique). Moreover, given subsets $W, W_{1}, \ldots, W_{n}$ of $X^{*}$ and a string $\omega \in X^{*}$, we write

$$
W_{1} \bowtie \cdots \bowtie W_{n}=\left\{\omega_{1} \bowtie \omega_{2} \bowtie \cdots \bowtie \omega_{n}: \omega_{i} \in W_{i}, 1 \leq i \leq n\right\}
$$

and

$$
\omega \bowtie W=\{\omega \bowtie \eta: \eta \in W\},
$$

where each symbol $\bowtie$ on the right-end sides runs over all admissible bridges. Finally, we write $W^{\bowtie n}=W_{1} \bowtie \cdots \bowtie W_{n}$ when $W_{1}=\cdots=$ $W_{n}=W$. 
2.2. Irregular sets. Let $\sigma: X \rightarrow X$ be a subshift. Given continuous functions $\varphi, \psi: X \rightarrow \mathbb{R}$ with $\psi>0$, we consider the level sets

$$
B_{\varphi, \psi}(\alpha)=\left\{\omega \in X: \lim _{n \rightarrow \infty} S_{\varphi, \psi}(\omega, n)=\alpha\right\},
$$

where

$$
S_{\varphi, \psi}(\omega, n)=\frac{\sum_{i=0}^{n-1} \varphi\left(\sigma^{i}(\omega)\right)}{\sum_{i=0}^{n-1} \psi\left(\sigma^{i}(\omega)\right)} .
$$

Moreover, we consider the open interval

$$
\mathcal{L}_{\varphi, \psi}=\left(\inf _{\mu} \frac{\int_{X} \varphi d \mu}{\int_{X} \psi d \mu}, \sup _{\mu} \frac{\int_{X} \varphi d \mu}{\int_{X} \psi d \mu}\right)
$$

where the infimum and supremum are taken over all $\sigma$-invariant probability measures on $X$. Given an interval $I \subset \mathcal{L}_{\varphi, \psi}$, we consider the irregular set

$$
X_{\varphi, \psi, I}=\left\{\omega \in X: A_{\varphi, \psi}(\omega)=I\right\},
$$

where $A_{\varphi, \psi}(\omega)$ is the set of accumulation points of the sequence $S_{\varphi, \psi}(\omega, n)$.

The following is our main result.

Theorem 3. Let $\sigma \mid X$ be a subshift with the weak specification property and let $\varphi, \psi: X \rightarrow \mathbb{R}$ be continuous function with inf $\psi>0$. Given a closed interval $I \subset \mathcal{L}_{\varphi, \psi}$ that is not a singleton, the set $X_{\varphi, \psi, I}$ is either empty or residual.

Proof: For each $\alpha \in \mathbb{R}, n \in \mathbb{N}$ and $\varepsilon>0$, let

$$
F(\alpha, n, \varepsilon)=\left\{\omega \mid n: \omega \in X \text { and }\left|\sum_{i=0}^{n-1} \varphi\left(\sigma^{i}(\omega)\right)-\alpha \sum_{i=0}^{n-1} \psi\left(\sigma^{i}(\omega)\right)\right|<n \varepsilon\right\} .
$$

It follows from Birkhoff's Ergodic Theorem that $F(\alpha, n, \varepsilon) \neq \varnothing$ for each $\alpha \in \mathcal{L}_{\varphi, \psi}$ and any sufficiently large $n$ (depending on $\alpha$ and $\varepsilon$ ). Take numbers $\alpha_{k, 1}, \ldots, \alpha_{k, q_{k}} \in I$ for $k \in \mathbb{N}$ such that

$$
I \subset \bigcup_{i=1}^{q_{k}} B\left(\alpha_{k, i}, 1 / k\right)
$$

and

$$
\left|\alpha_{k, i+1}-\alpha_{k, i}\right|<\frac{1}{k} \text { for } i=0, \ldots, q_{k}-1, \quad\left|\alpha_{k, q_{k}}-\alpha_{k+1,1}\right|<\frac{1}{k} .
$$


Moreover, let $\varepsilon_{1}>\varepsilon_{2}>\cdots$ be a sequence of positive numbers decreasing to zero and let

$$
n_{1,1}<n_{1,2}<\cdots<n_{1, q_{1}}<n_{2,1}<n_{2,2}<\cdots<n_{2, q_{2}}<\cdots
$$

be positive integers such that

$$
F_{k, i}:=F\left(\alpha_{k, i}, n_{k, i}, \varepsilon_{k}\right) \neq \varnothing \quad \text { for } \quad k \in \mathbb{N}, 1 \leq i \leq q_{k} .
$$

Let $\Omega_{0}=X^{*}$. For each $\omega \in \Omega_{0}$, take integers $\left\{N_{k, i}(\omega)\right\}_{k \in \mathbb{N}, i=1, \ldots, q_{k}}$ such that:

(i) $N_{1, i}(\omega) \geq 2^{n_{1, i+1}+\tau}$ for $2 \leq i \leq q_{1}-1$,

$N_{k, i}(\omega) \geq 2^{n_{k, i+1}+\tau}$ for $k \geq 2$ and $1 \leq i \leq q_{k}-1$,

$N_{k, q_{k}}(\omega) \geq 2^{n_{k+1,1}+\tau}$ for $k \geq 1$;

(ii) $N_{k, i+1}(\omega) \geq 2^{|\omega|+\tau+N_{1,1}(\omega)\left(n_{1,1}+\tau\right)+N_{1,2}(\omega)\left(n_{1,2}+\tau\right)+\cdots+N_{k, i}(\omega)\left(n_{k, i}+\tau\right)}$,

$N_{k+1,1}(\omega) \geq 2^{|\omega|+\tau+N_{1,1}(\omega)\left(n_{1,1}+\tau\right)+N_{1,2}(\omega)\left(n_{1,2}+\tau\right)+\cdots+N_{k, q_{k}}(\omega)\left(n_{k, q_{k}}+\tau\right)}$

for $k \in \mathbb{N}$ and $i=1, \ldots, q_{k}-1$.

Here $\tau$ is the integer in the notion of weak specification. Now we define recursively sets $\Omega_{k, i} \subset X^{*}$ for $k \in \mathbb{N}$ and $i=1, \ldots, q_{k}$ by

$$
\begin{aligned}
\Omega_{1,1} & =\bigcup_{\omega \in \Omega_{0}} \omega \bowtie\left(F_{1,1}\right)^{\bowtie N_{1,1}(\omega),} \\
\Omega_{1,2} & =\bigcup_{\eta \in \Omega_{1,1}} \eta \bowtie\left(F_{1,2}\right)^{\bowtie N_{1,2}(\omega),} \\
& \vdots \\
\Omega_{1, q_{1}} & =\bigcup_{\eta \in \Omega_{1, q_{1}-1}} \eta \bowtie\left(F_{1, q_{1}}\right)^{\bowtie N_{1, q_{1}}(\omega),} \\
\Omega_{2,1} & =\bigcup_{\eta \in \Omega_{1, q_{1}}} \eta \bowtie\left(F_{2,1}\right)^{\bowtie N_{2,1}(\omega),}
\end{aligned}
$$

and so on. Finally, let

$$
E_{k, i}=\bigcup_{\omega \in \Omega_{k, i}}[\omega]
$$

and consider the $G_{\delta}$ set

$$
E=\bigcap_{k=1}^{\infty} \bigcap_{i=1}^{q_{k}} E_{k, i} .
$$

Since $\Omega_{0}=X^{*}$, each set $E_{k, i}$ is dense and it follows from Baire's Theorem that $E$ is also dense. 
In order to prove that $E \subset X_{\varphi, \psi, I}$, we must show that $A_{\varphi, \psi}(\omega)=I$ for $\omega \in E$. We first show that

$$
I \subset A_{\varphi, \psi}(\omega) .
$$

For each $\omega \in E$, take $\omega^{0} \in \Omega_{0}$ such that

$$
\omega \in \omega^{0} \bowtie\left(F_{1,1}\right)^{\bowtie N_{1,1}\left(\omega^{0}\right)} \bowtie \cdots .
$$

Given $\alpha \in I$, take an integer $i_{k} \in\left\{1, \ldots, q_{k}\right\}$ such that $\alpha \in B\left(\alpha_{k, i_{k}}, 1 / k\right)$. For simplicity of the notation we assume that $i_{k} \notin\left\{1, q_{k}\right\}$ although the argument is identical for $i_{k} \in\left\{1, q_{k}\right\}$. Let

(6) $s_{k, i_{k}}=\left|\omega^{0}\right|+\lambda_{0}+\sum_{j=1}^{q_{1}} \sum_{l=1}^{N_{1, j}}\left(n_{1, j}+\lambda_{1, j, l}\right)+\cdots+\sum_{j=1}^{i_{k}} \sum_{l=1}^{N_{k, j}}\left(n_{k, j}+\lambda_{k, j, l}\right)$,

where $\lambda_{0}$ is the length of the first bridge, $N_{k, j}=N_{k, j}\left(\omega^{0}\right)$ and

$$
\lambda_{i, j, l}= \begin{cases}\tau_{i, j} & \text { if } l \neq N_{i, j} \\ \tau_{i, j}^{\prime} & \text { if } l=N_{i, j}\end{cases}
$$

is the length of each successive bridge between strings of $\omega^{0}$. By the weak specification property, the numbers $\lambda_{0}$ and $\lambda_{i, j, l}$ are bounded by $\tau$.

Write

$$
s_{k, i_{k}}=\widetilde{s}_{k, i_{k}}+t_{k, i_{k}},
$$

where

$$
t_{k, i_{k}}=\sum_{l=1}^{N_{k, i_{k}}}\left(n_{k, i_{k}}+\lambda_{k, i_{k}, l}\right)
$$

Since $M:=\sup _{k}\left|\alpha_{k, i_{k}}\right|<\infty$, writing

$$
c=\|\varphi\|+M\|\psi\| \quad \text { and } \quad r_{l}=\widetilde{s}_{k, i_{k}}+(l-1)\left(n_{k, i_{k}}+\lambda_{k, i_{k}, l}\right),
$$


we obtain

(8)

$$
\begin{aligned}
& \left|\sum_{i=0}^{s_{k, i_{k}}-1} \varphi\left(\sigma^{i}(\omega)\right)-\sum_{i=0}^{s_{k, i_{k}}-1} \psi\left(\sigma^{i}(\omega)\right) \alpha_{k, i_{k}}\right| \\
& \leq\left|\sum_{i=0}^{\widetilde{s}_{k, i_{k}}-1} \varphi\left(\sigma^{i}(\omega)\right)-\sum_{i=0}^{\widetilde{s}_{k, i_{k}}-1} \psi\left(\sigma^{i}(\omega)\right) \alpha_{k, i_{k}}\right| \\
& +\left|\sum_{i=\widetilde{s}_{k, i_{k}}}^{s_{k, i_{k}}-1} \varphi\left(\sigma^{i}(\omega)\right)-\sum_{i=\widetilde{s}_{k, i_{k}}}^{s_{k, i_{k}}-1} \psi\left(\sigma^{i}(\omega)\right) \alpha_{k, i_{k}}\right| \\
& \leq \widetilde{s}_{k, i_{k}} c+\left|\sum_{i=0}^{t_{k, i_{k}}-1} \varphi\left(\sigma^{i}\left(\sigma^{\widetilde{s}_{k, i_{k}}}(\omega)\right)\right)-\sum_{i=0}^{t_{k, i_{k}}-1} \psi\left(\sigma^{i}\left(\sigma^{\widetilde{s}_{k, i_{k}}}(\omega)\right)\right) \alpha_{k, i_{k}}\right| \\
& \leq \widetilde{s}_{k, i_{k}} c+\sum_{l=1}^{N_{k, i_{k}}}\left|\sum_{j=0}^{n_{k, i_{k}}-1} \varphi\left(\sigma^{j}\left(\sigma^{r_{l}}(\omega)\right)\right)-\sum_{j=0}^{n_{k, i_{k}}-1} \psi\left(\sigma^{j}\left(\sigma^{r_{l}}(\omega)\right)\right) \alpha_{k, i_{k}}\right| \\
& +\sum_{l=1}^{N_{k, i_{k}}}\left|\sum_{j=n_{k, i_{k}}}^{n_{k, i_{k}}+\lambda_{i, i_{k}, l}-1} \varphi\left(\sigma^{j}\left(\sigma^{r_{l}}(\omega)\right)\right)-\sum_{j=n_{k, i_{k}}}^{n_{k, i_{k}}+\lambda_{i, i_{k}, l}-1} \psi\left(\sigma^{j}\left(\sigma^{r_{l}}(\omega)\right)\right) \alpha_{k, i_{k}}\right| \\
& \leq \widetilde{s}_{k, i_{k}} c+\sum_{l=1}^{N_{k, i_{k}}}\left|\sum_{j=0}^{n_{k, i_{k}}-1} \varphi\left(\sigma^{j}\left(\sigma^{r_{l}}(\omega)\right)\right)-\sum_{j=0}^{n_{k, i_{k}}-1} \psi\left(\sigma^{j}\left(\sigma^{r_{l}}(\omega)\right)\right) \alpha_{k, i_{k}}\right| \\
& +\tau N_{k, i_{k}} c .
\end{aligned}
$$

In order to estimate the second term in the right-hand side of (8), we introduce the numbers

$$
v_{n}(\varphi)=\sup \left\{\left|\varphi(\omega)-\varphi\left(\omega^{\prime}\right)\right|: \omega, \omega^{\prime} \in X, \omega\left|n=\omega^{\prime}\right| n\right\}
$$

and $V_{n}(\varphi)=\sum_{j=1}^{n} v_{j}(\varphi)$.

By (5) and the definition of the set $F_{k, i_{k}}$, there exist $\bar{\omega}^{1}, \ldots, \bar{\omega}^{N_{k, i_{k}}} \in$ $X$ such that

$$
\sigma^{\widetilde{s}_{k, i_{k}}+(l-1)\left(n_{k, i_{k}}+\lambda_{k, i_{k}, l}\right)}(\omega)\left|n_{k, i_{k}}=\bar{\omega}^{l}\right| n_{k, i_{k}}
$$


and

$$
\left|\sum_{i=0}^{n_{k, i_{k}}-1} \varphi\left(\sigma^{i}\left(\bar{\omega}^{l}\right)\right)-\alpha_{k, i_{k}} \sum_{i=0}^{n_{k, i_{k}}-1} \psi\left(\sigma^{i}\left(\bar{\omega}^{l}\right)\right)\right|<n_{k, i_{k}} \varepsilon_{k}
$$

for $l=1, \ldots, N_{k, i_{k}}$. It follows from (9) and (10) that

$$
\begin{aligned}
& \left|\sum_{j=0}^{n_{k, i_{k}}-1} \varphi\left(\sigma^{j}\left(\sigma^{\widetilde{s}_{k, i_{k}}+(l-1) r_{l}}(\omega)\right)\right)-\sum_{j=0}^{n_{k, i_{k}}-1} \psi\left(\sigma^{j}\left(\sigma^{\widetilde{s}_{k, i_{k}}+(l-1) r_{l}}(\omega)\right)\right) \alpha_{k, i_{k}}\right| \\
& \leq\left|\sum_{j=0}^{n_{k, i_{k}}-1} \varphi\left(\sigma^{j}\left(\sigma^{\widetilde{s}_{k, i_{k}}+(l-1) r_{l}}(\omega)\right)\right)-\sum_{j=0}^{n_{k, i_{k}}-1} \varphi\left(\sigma^{j}\left(\bar{\omega}^{l}\right)\right)\right| \\
& \quad+\left|\sum_{j=0}^{n_{k, i_{k}}-1} \varphi\left(\sigma^{j}\left(\bar{\omega}^{l}\right)\right)-\alpha_{k, i_{k}} \sum_{j=0}^{n_{k, i_{k}}-1} \psi\left(\sigma^{j}\left(\bar{\omega}^{l}\right)\right)\right| \\
& \quad+\left|\sum_{j=0}^{n_{k, i_{k}}-1} \psi\left(\sigma^{j}\left(\bar{\omega}^{l}\right)\right)-\sum_{j=0}^{n_{k, i_{k}}-1} \psi\left(\sigma^{j}\left(\sigma^{\widetilde{s}_{k, i_{k}}}+(l-1) r_{l}(\omega)\right)\right)\right| \alpha_{k, i_{k}} \\
& \leq
\end{aligned}
$$

for $l=1, \ldots, N_{k, i_{k}}$. Together with (8) this implies that

$$
\begin{aligned}
& \left|\sum_{i=0}^{s_{k, i_{k}}-1} \varphi\left(\sigma^{i}(\omega)\right)-\sum_{i=0}^{s_{k, i_{k}}-1} \psi\left(\sigma^{i}(\omega)\right) \alpha_{k, i_{k}}\right| \\
& \leq \widetilde{s}_{k, i_{k}} c+N_{k, i_{k}}\left(V_{n_{k, i_{k}}}(\varphi)+n_{k, i_{k}} \varepsilon_{k}+M V_{n_{k, i_{k}}}(\psi)\right)+\tau N_{k, i_{k}} c \\
& =\widetilde{s}_{k, i_{k}} c+N_{k, i_{k}}\left(V_{n_{k, i_{k}}}(\varphi)+M V_{n_{k, i_{k}}}(\psi)\right)+N_{k, i_{k}}\left(n_{k, i_{k}} \varepsilon_{k}+\tau c\right) .
\end{aligned}
$$

Now we observe that it follows from condition (ii) that $\widetilde{s}_{k, i_{k}} / s_{k, i_{k}} \rightarrow 0$ when $k \rightarrow \infty$ : using (6), (7) and condition (ii), we have

$$
\frac{s_{k, i_{k}}}{\widetilde{s}_{k, i_{k}}}-1=\frac{t_{k, i_{k}}}{\widetilde{s}_{k, i_{k}}} \geq \frac{N_{k, i_{k}}}{\widetilde{s}_{k, i_{k}}} n_{k, i_{k}} \geq \frac{2^{\widetilde{s}_{k, i_{k}}}}{\widetilde{s}_{k, i_{k}}} n_{k, i_{k}}
$$

and thus, $s_{k, i_{k}} / \widetilde{s}_{k, i_{k}} \rightarrow+\infty$ when $k \rightarrow \infty$. On the other hand, it follows from the uniform continuity of $\varphi$ on the compact set $X$ that $v_{n}(\varphi) \rightarrow 0$ when $n \rightarrow \infty$. Hence, $V_{n}(\varphi) / n \rightarrow 0$ when $n \rightarrow \infty$ and

$$
\frac{N_{k, i_{k}}\left(V_{n_{k, i_{k}}}(\varphi)+M V_{n_{k, i_{k}}}(\psi)\right)}{s_{k, i_{k}}} \leq \frac{V_{n_{k, i_{k}}}(\varphi)}{n_{k, i_{k}}}+\frac{M V_{n_{k, i_{k}}}(\psi)}{n_{k, i_{k}}} \rightarrow 0
$$


when $k \rightarrow \infty$. Finally, by the definition of $s_{k, i_{k}}$, we have $s_{k, i_{k}}>t_{k, i_{k}}$ and

Therefore,

$$
\frac{N_{k, i_{k}} n_{k, i_{k}} \varepsilon_{k}}{s_{k, i_{k}}}<\frac{N_{k, i_{k}} n_{k, i_{k}} \varepsilon_{k}}{t_{k, i_{k}}} \leq \varepsilon_{k} .
$$

$$
\begin{aligned}
\mid S_{\varphi, \psi}\left(\omega, s_{k, i_{k}}\right) & -\alpha_{k, i_{k}} \mid \\
< & \frac{\widetilde{s}_{k, i_{k}}}{s_{k, i_{k}}} c+\frac{V_{n_{k, i_{k}}}(\varphi)+M V_{n_{k, i_{k}}}(\psi)+n_{k, i_{k}} \varepsilon_{k}+\tau c}{n_{k, i_{k}} \inf \psi} \rightarrow 0
\end{aligned}
$$

when $k \rightarrow \infty$. This implies that

$$
\begin{aligned}
\left|S_{\varphi, \psi}\left(\omega, s_{k, i_{k}}\right)-\alpha\right| & \leq\left|S_{\varphi, \psi}\left(\omega, s_{k, i_{k}}\right)-\alpha_{k, i_{k}}\right|+\left|\alpha_{k, i_{k}}-\alpha\right| \\
& <\left|S_{\varphi, \psi}\left(\omega, s_{k, i_{k}}\right)-\alpha_{k, i_{k}}\right|+\frac{1}{k} \rightarrow 0
\end{aligned}
$$

when $k \rightarrow \infty$. Hence, $\alpha \in A_{\varphi, \psi}(\omega)$ and (4) holds.

Now we show that

$$
A_{\varphi, \psi}(\omega) \subset I .
$$

For each positive integer $n>\left|\omega^{0}\right|+\tau$ there exist $k \in \mathbb{N}, i_{k} \in$ $\left\{1,2, \ldots, q_{k}\right\}$ and $1 \leq p \leq N_{k, i_{k}+1}$ such that

$$
s_{k, i_{k}}+\rho_{p}<n \leq s_{k, i_{k}}+\rho_{p+1}
$$

where

$$
\rho_{s}=\sum_{l=1}^{s-1}\left(n_{k, i_{k}+1}+\lambda_{k, i_{k}+1, l}\right)
$$

for $s \in \mathbb{N}$. Notice that $k \rightarrow \infty$ when $n \rightarrow \infty$. We have

$$
\begin{aligned}
& \left|\sum_{i=0}^{n-1} \varphi\left(\sigma^{i}(\omega)\right)-\sum_{i=0}^{n-1} \psi\left(\sigma^{i}(\omega)\right) \alpha_{k, i_{k}}\right| \\
& \leq\left|\sum_{i=0}^{s_{k, i_{k}}-1} \varphi\left(\sigma^{i}(\omega)\right)-\sum_{i=0}^{s_{k, i_{k}}-1} \psi\left(\sigma^{i}(\omega)\right) \alpha_{k, i_{k}}\right| \\
& +\left|\sum_{i=s_{k, i_{k}}}^{s_{k, i_{k}}+\rho_{p}-1} \varphi\left(\sigma^{i}(\omega)\right)-\sum_{i=s_{k, i_{k}}}^{s_{k, i_{k}}+\rho_{p}-1} \psi\left(\sigma^{i}(\omega)\right) \alpha_{k, i_{k}}\right| \\
& +\left|\sum_{i=s_{k, i_{k}}+\rho_{p}}^{n-1} \varphi\left(\sigma^{i}(\omega)\right)-\sum_{i=s_{k, i_{k}}+\rho_{p}}^{n-1} \psi\left(\sigma^{i}(\omega)\right) \alpha_{k, i_{k}}\right| .
\end{aligned}
$$


Similarly, one can choose $\bar{\omega}^{1}, \ldots, \bar{\omega}^{p-1} \in X$ such that

$$
\sigma^{s_{k, i_{k}}+(l-1)\left(n_{k, i_{k}+1}+\lambda_{k, i_{k}+1, l}\right)}(\omega)\left|n_{k, i_{k}+1}=\bar{\omega}^{l}\right| n_{k, i_{k}+1}
$$

and

$$
\left|\sum_{i=0}^{n_{k, i_{k}+1}-1} \varphi\left(\sigma^{i}\left(\bar{\omega}^{l}\right)\right)-\alpha_{k, i_{k}+1} \sum_{i=0}^{n_{k, i_{k}+1}-1} \psi\left(\sigma^{i}\left(\bar{\omega}^{l}\right)\right)\right|<n_{k, i_{k}+1} \varepsilon_{k}
$$

for $l=1, \ldots, p-1$. Writing $r_{l}=s_{k, i_{k}}+(l-1)\left(n_{k, i_{k}+1}+\lambda_{k, i_{k}+1, l}\right)$, it follows from (3), (14) and (15) that

$$
\begin{aligned}
& \left|\sum_{j=0}^{n_{k, i_{k}+1}-1} \varphi\left(\sigma^{j}\left(\sigma^{r_{l}}(\omega)\right)\right)-\sum_{j=0}^{n_{k, i_{k}+1}-1} \psi\left(\sigma^{j}\left(\sigma^{r_{l}}(\omega)\right)\right) \alpha_{k, i_{k}}\right| \\
& \leq\left|\sum_{j=0}^{n_{k, i_{k}+1}-1} \varphi\left(\sigma^{j}\left(\sigma^{r_{l}}(\omega)\right)\right)-\sum_{j=0}^{n_{k, i_{k}+1}-1} \psi\left(\sigma^{j}\left(\sigma^{r_{l}}(\omega)\right)\right) \alpha_{k, i_{k}+1}\right| \\
& +\left|\sum_{j=0}^{n_{k, i_{k}+1}-1} \psi\left(\sigma^{j}\left(\sigma^{r_{l}}(\omega)\right)\right) \alpha_{k, i_{k}+1}-\sum_{j=0}^{n_{k, i_{k}+1}-1} \psi\left(\sigma^{j}\left(\sigma^{r_{l}}(\omega)\right)\right) \alpha_{k, i_{k}}\right| \\
& \leq\left|\sum_{j=0}^{n_{k, i_{k}+1}-1} \varphi\left(\sigma^{j}\left(\sigma^{r_{l}}(\omega)\right)\right)-\sum_{j=0}^{n_{k, i_{k}+1}-1} \varphi\left(\sigma^{j}\left(\bar{\omega}^{l}\right)\right)\right| \\
& +\mid \sum_{j=0}^{n_{k, i_{k}+1}-1} \varphi\left(\sigma^{j}\left(\bar{\omega}^{l}\right)-\sum_{j=0}^{n_{k, i_{k}+1}-1} \psi\left(\sigma^{j}\left(\bar{\omega}^{l}\right) \alpha_{k, i_{k}+1} \mid\right.\right. \\
& +\left|\sum_{j=0}^{n_{k, i_{k}+1}-1} \psi\left(\sigma^{j}\left(\bar{\omega}^{l}\right)\right)-\sum_{j=0}^{n_{k, i_{k}+1}-1} \psi\left(\sigma^{j}\left(\sigma^{r_{l}}(\omega)\right)\right)\right| \alpha_{k, i_{k}+1}+\frac{n_{k, i_{k}+1}\|\psi\|}{k} \\
& \leq V_{n_{k, i_{k}+1}}(\varphi)+n_{k, i_{k}+1} \varepsilon_{k}+M V_{n_{k, i_{k}+1}}(\psi)+\frac{n_{k, i_{k}+1}\|\psi\|}{k}
\end{aligned}
$$

for $l=1, \ldots, p-1$. 
Therefore,

$$
\begin{aligned}
& \left|\sum_{i=s_{k, i_{k}}}^{s_{k, i_{k}}+\rho_{p}-1} \varphi\left(\sigma^{i}(\omega)\right)-\sum_{i=s_{k, i_{k}}}^{s_{k, i_{k}}+\rho_{p}-1} \psi\left(\sigma^{i}(\omega)\right) \alpha_{k, i_{k}}\right| \\
& \leq\left|\sum_{l=1}^{p-1}\left(\sum_{j=0}^{n_{k, i_{k}+1}-1} \varphi\left(\sigma^{j}\left(\sigma^{r_{l}}(\omega)\right)\right)-\sum_{j=0}^{n_{k, i_{k}+1}-1} \psi\left(\sigma^{j}\left(\sigma^{r_{l}}(\omega)\right)\right) \alpha_{k, i_{k}}\right)\right|+(p-1) \tau c \\
& \leq(p-1)\left(V_{n_{k, i_{k}+1}}(\varphi)+M V_{n_{k, i_{k}+1}}(\psi)\right) \\
& \quad+(p-1)\left(n_{k, i_{k}+1} \varepsilon_{k}+\tau c\right)+\frac{(p-1) n_{k, i_{k}+1}\|\psi\|}{k} .
\end{aligned}
$$

Moreover, by (13), we have

$$
\begin{aligned}
\left|\sum_{i=s_{k, i_{k}}+\rho_{p}}^{n-1} \varphi\left(\sigma^{i}(\omega)\right)-\sum_{i=s_{k, i_{k}}+\rho_{p}}^{n-1} \psi\left(\sigma^{i}(\omega)\right) \alpha_{k, i_{k}}\right| & \leq\left(n-s_{k, i_{k}}-\rho_{p}\right) c \\
& \leq\left(n_{k, i_{k}+1}+\tau\right) c .
\end{aligned}
$$

Therefore,

$$
\begin{aligned}
\left|S_{\varphi, \psi}(\omega, n)-\alpha_{k, i_{k}}\right| \leq & \left|S_{\varphi, \psi}\left(\omega, s_{k, i_{k}}\right)-\alpha_{k, i_{k}}\right| \\
& +\frac{(p-1)}{n \inf \psi}\left(V_{n_{k, i_{k}+1}}(\varphi)+M V_{n_{k, i_{k}+1}}(\psi)\right) \\
& +\frac{(p-1)}{n \inf \psi}\left(n_{k, i_{k}+1} \varepsilon_{k}+\tau c\right) \\
& +\frac{(p-1) n_{k, i_{k}+1}\|\psi\|}{k n \inf \psi}+\frac{\left(n_{k, i_{k}+1}+\tau\right) c}{n \inf \psi} .
\end{aligned}
$$

In a similar manner to that in (11), one can show that the first term in (16) tends to zero when $n \rightarrow \infty$ (notice that $s_{k, i_{k}} \leq n$ ). Moreover, using (13) and condition (i), we obtain

$$
\frac{\left(n_{k, i_{k}+1}+\tau\right)}{n} \leq \frac{\left(n_{k, i_{k}+1}+\tau\right)}{s_{k, i_{k}}} \leq \frac{\left(n_{k, i_{k}+1}+\tau\right)}{N_{k, i_{k}}} \rightarrow 0
$$


when $n \rightarrow \infty$. On the other hand, it follows from (13) that

$$
\begin{gathered}
\frac{(p-1) n_{k, i_{k}+1}}{k n} \leq \frac{1}{k} \rightarrow 0 \quad \text { when } n \rightarrow \infty \\
\frac{(p-1)\left(V_{n_{k, i_{k}+1}}(\varphi)+V_{n_{k, i_{k}+1}}(\psi)\right)}{n} \leq \frac{V_{n_{k, i_{k}+1}}(\varphi)}{n_{k, i_{k}+1}}+\frac{V_{n_{k, i_{k}+1}}(\psi)}{n_{k, i_{k}+1}} \rightarrow 0
\end{gathered}
$$

when $n \rightarrow \infty$, and

$$
\frac{(p-1) n_{k, i_{k}+1} \varepsilon_{k}}{n} \leq \frac{n_{k, i_{k}+1} \varepsilon_{k}}{n_{k, i_{k}+1}} \rightarrow 0 \quad \text { when } \quad n \rightarrow \infty
$$

(since $k \rightarrow \infty$ when $n \rightarrow \infty$ ).

By (16), (17), (18), (19), and (20), we obtain

$$
\left|S_{\varphi, \psi}(\omega, n)-\alpha_{k, i_{k}}\right| \rightarrow 0 \quad \text { when } n \rightarrow \infty .
$$

It follows from (2) that

$$
\operatorname{dist}\left(S_{\varphi, \psi}(\omega, n), I\right) \leq\left|S_{\varphi, \psi}(\omega, n)-\alpha_{k, i_{k}}\right|+\operatorname{dist}\left(\alpha_{k, i_{k}}, I\right) \rightarrow 0
$$

when $k \rightarrow \infty$. Since $I$ is closed, this yields inclusion (12). Therefore, $E \subset X$ is a dense $G_{\delta}$ set such that $E \subset X_{\varphi, \psi, I}$. This completes the proof of the theorem.

\section{References}

[1] L. BARReIRA, "Dimension and recurrence in hyperbolic dynamics", Progress in Mathematics 272 , Birkhäuser Verlag, Basel, 2008.

[2] L. Barreira, J. Li, ANd C. VAlls, Irregular sets are residual, Preprint.

[3] L. Barreira and B. SAussol, Multifractal analysis of hyperbolic flows, Comm. Math. Phys. 214(2) (2000), 339-371.

[4] L. Barreira and J. Schmeling, Sets of "non-typical" points have full topological entropy and full Hausdorff dimension, Israel $J$. Math. 116 (2000), 29-70. DOI: 10.1007/BF02773211.

[5] C. Ercai, T. Küpper, and S. Lin, Topological entropy for divergence points, Ergodic Theory Dynam. Systems 25(4) (2005), 1173-1208. DOI : 10.1017/S0143385704000872.

[6] A.-H. Fan, D.-J. Feng, And J. Wu, Recurrence, dimension and entropy, J. London Math. Soc. (2) 64(1) (2001), 229-244. DOI: 10.1017/S0024610701002137.

[7] D.-J. Feng, K.-S. LaU, and J. Wu, Ergodic limits on the conformal repellers, Adv. Math. 169(1) (2002), 58-91. DOI: 10.1006 /aima.2001.2054. 
[8] J. LI AND M. WU, Divergence points in systems satisfying the specification property, Discrete Contin. Dyn. Syst. 33(2) (2013), 905-920.

[9] Ya. B. Pesin And B. S. Pitskel', Topological pressure and the variational principle for noncompact sets, (Russian), Funktsional. Anal. i Prilozhen. 18(4) (1984), 50-63, 96.

[10] D. Thompson, The irregular set for maps with the specification property has full topological pressure, Dyn. Syst. 25(1) (2010), 25-51. DOI: 10.1080/14689360903156237.

Luis Barreira and Claudia Valls:

Departamento de Matemática

Instituto Superior Técnico

1049-001 Lisboa

Portugal

E-mail address: barreira@math.ist.utl.pt

E-mail address: cvalls@math.ist.utl.pt

Jinjun Li:

School of Mathematics and Statistics

Minnan Normal University

Zhangzhou, 363000

P. R. China

E-mail address: li-jinjun@163.com 